\title{
Estabilização da biomassa de aguapé através da compostagem com águas resíduárias de suínos e resíduos de frigorífico ${ }^{1}$
}

\author{
Water hyacinth biomass stabilization by its composting with swine \\ wasterwater and slaugther house wasters
}

\author{
Juliana Bortoli Rodrigues Mees ${ }^{2 *}$; Simone Damasceno ${ }^{3}$; \\ Márcio Antônio Vilas Boas ${ }^{4}$; Ajadir Fazolo ${ }^{5}$; Silvio César Sampaio ${ }^{6}$
}

\begin{abstract}
Resumo
O presente trabalho teve por objetivo avaliar a compostagem da biomassa de aguapé (E. crassipes), após sua utilização na remoção de poluentes em sistema de tratamento de efluente de uma Unidade Frigorífica de suínos, com o aproveitamento de águas residuárias provenientes da higienização de pocilgas e dos caminhões que transportam os suínos, e das tripas celulósicas geradas no processamento de salsichas. Avaliou-se a compostagem, montando-se oito leiras com aproximadamente $0,60 \mathrm{~m}^{3}$, com quatro tratamentos distintos e duas repetições, sendo: $\mathrm{T}_{1}-$ Aguapé (E. crassipes), $\mathrm{T}_{2}-$ Aguapé e dejeto suíno, $\mathrm{T}_{3}-$ Aguapé, dejeto suíno e terra, $\mathrm{T}_{4}$ - Aguapé, dejeto suíno e tripa celulósica, por um período de 90 dias. Considerando a relação C:N como um indicador da maturidade do composto, observou-se que o tratamento T4 (aguapé, dejeto suíno e tripa celulósica) obteve o menor período de estabilização, 60 dias. Com relação à taxa de bioestabilização a análise estatística mostrou que não houve diferença significativa ao nível 5\% de probabilidade pelo teste $\mathrm{F}$ para os quatro tratamentos avaliados no período de 90 dias. A taxa de bioestabilização média para o carbono orgânico total e para o nitrogênio total foram, respectivamente, $1,8 \times 10^{-2} \mathrm{dia}^{-1}$ e $0,8 \times 10^{-3} \mathrm{dia}^{-1}$.

Palavras-chave: Plantas aquáticas, composto orgânico, relação C:N
\end{abstract}

\begin{abstract}
The objective of the present research was to evaluate the composting of water hyacinth (E. crassipes) biomass, after its usage for the removal of pollutants from the effluent of a swine slaughter house wastewater treatment system, recycling the waste water used for swine transportation trucks and piggery hygienic cleaning and the cellulosic gut generated in the sausage processing. The composting was evaluated by building eight composting piles measuring approximately $0,60 \mathrm{~m}^{3}$ each; the piles
\end{abstract}

\footnotetext{
1 Parte da dissertação de mestrado do primeiro autor. Experimento desenvolvido em um abatedouro e frigorífico de suínos, localizado na região oeste do Estado do Paraná.

2 Professora da Universidade Tecnológica Federal do Paraná/UTFPR, Doutoranda do Programa de pós-graduação em Engenharia Agrícola: Recursos Hídricos e Saneamento Ambiental, da Universidade Estadual do Oeste do Paraná/UNIOESTE, Mestre em Engenharia Agrícola: Recursos Hídricos e Meio ambiente, Graduada em Tecnologia Ambiental: Resíduos Industriais. E-mail: juliana@utfpr.edu.br; julianabortolimees@hotmail.com

3 Engenheira Agrônoma, Doutora em Agronomia, Professora da Universidade Estadual do Oeste do Paraná/UNIOESTE. E-mail: simoned@unioeste.br

4 Engenheiro Agrícola, Doutor em Agronomia, Professor da Universidade Estadual do Oeste do Paraná/UNIOESTE. E-mail: vilasma@unioeste.br

5 Engenheiro Sanitarista, Doutor em Engenharia Hidráulica e Saneamento, Professor da Universidade Tecnológica Federal do Paraná/UTFPR. E-mail: afazolo@utfpr.edu.br

6 Engenheiro Agrícola, Doutor em Engenharia Agrícola, Professor da Universidade Estadual do Oeste do Paraná/UNIOESTE. E-mail: ssampaio@unioeste.br

* Autor para correspondência
} 
consisted of four distinct treatments that were done twice. The treatments were: $\mathrm{T}_{1}-$ Water hyacinth $(E$. crassipes), $\mathrm{T}_{2}$ - Water hyacinth and swine excrement, $\mathrm{T}_{3}-$ Water hyacinth, swine excrement and earth, $\mathrm{T}_{4}$ - Water hyacinth, swine excrement and cellulosic gut, for a period of 90 days. Considering the C:N ratio as a compost maturity indicator, it was observed that the T4 treatment (water hyacinth, excrement and cellulosic gut) had the shorter period of stabilization, 60 days. Regarding the biostabilization rate, the statistic analysis showed that there was no significant difference at $5 \%$ level by the $\mathrm{F}$ test between the four treatments evaluated during 90 days. The total organic carbon and the nitrogen biostabilization average rates were $1,8 \times 10^{-2} \mathrm{day}^{-1}$ and $0,8 \times 10^{-3} \mathrm{day}^{-1}$, respectively.

Key words: Aquatic plants, organic compost, $\mathrm{C}: \mathrm{N}$ ratio

\section{Introdução}

Mundialmente a demanda de água está crescendo rapidamente à medida que a população, a atividade industrial e a agricultura irrigada se expandem. Os processos industriais, apesar de ocuparem a segunda posição no consumo total de água com $22 \%$, são um dos principais responsáveis pela poluição das águas, quando lançam efluentes sem tratamento adequado aos corpos d'água. Dentro do setor industrial destacam-se as agroindústrias como grandes poluidoras, devido especialmente às elevadas vazões com que geram seus efluentes ricos em carga orgânica, nutrientes, sólidos e óleos e graxas.

De acordo com Grady, Daigger e Lim (1999), esses poluentes podem ser removidos por processos físicos, químicos e/ou bioquímicos. Porém, nem sempre estes processos convencionais conseguem remover a níveis aceitáveis, pela legislação vigente, a concentração de nutrientes e da matéria orgânica solúvel. Sendo necessário assim a aplicação de tratamentos avançados e/ou terciários para $\mathrm{o}$ polimento final, que muitas vezes são onerosos.

Segundo Lima et al. (2003), uma alternativa seria a utilização de plantas aquáticas e sua microbiota com a finalidade de remover, degradar ou isolar substâncias tóxicas do ambiente. No entanto para que as macrófitas aquáticas mantenham a eficiência na remoção da carga orgânica e de nutrientes das águas residuárias é necessária a manutenção da densidade adequada de plantas, através da remoção da biomassa em excesso e em estado de senescência.
A biomassa de aguapé, depois de removida do sistema de tratamento poderá ser utilizada para compostagem, e as leiras entrepostas com dejeto suíno e terra rica em húmus (WOLVERTON; MCDONALD, 1979; MORAES et al., 2002).

Suzek et al. (2005) afirmam a possibilidade da utilização de águas residuárias da suinocultura como inóculo no processo de bioestabilização de resíduos verdes urbanos, visando a obtenção subsequente de um vermicomposto.

Neste contexto o presente estudo teve como objetivo avaliar a compostagem da biomassa de aguapé (E. crassipes), removida do sistema de tratamento de efluente de uma Unidade Frigorífica, aproveitando no processo de compostagem outros resíduos anteriormente descartados pela indústria, tais comoaságuas residuárias geradas nahigienização das pocilgas e dos caminhões que transportam os suínos, assim como das tripas celulósicas geradas durante o processo de industrialização da salsicha.

\section{Material e métodos}

\section{Obtenção da biomassa de aguapé (E. Crassipes)}

O experimento foi instalado em uma Unidade Frigorífica localizada no município de Medianeira, Oeste do Estado do Paraná. Na Unidade são abatidos e industrializados diariamente cerca de 1500 suínos, gerando diariamente $1350 \mathrm{~m}^{3}$ de despejos líquidos, que são encaminhados ao sistema de tratamento composto por cinco células de tratamento, em série. 
Na quarta célula do sistema, lagoa de decantação, foram introduzidos aguapés numa área delimitada de $870 \mathrm{~m}^{2}$, com o objetivo de avaliar o desempenho da macrófita aquática na remoção de matéria-orgânica e de nutrientes do efluente líquido da indústria.

Durante os 11 meses de acompanhamento do sistema, controlou-se a densidade das plantas através de retiradas periódicas da biomassa em excesso.

A remoção foi efetuada de forma manual, com garfos coletores, e quando necessário com auxílio de um barco. A biomassa removida foi encaminhada para estabilização via processo de compostagem.

Compostagem da biomassa de aguapé (E. crassipes)

A compostagem foi efetuada em escala piloto em uma área delimitada de $50 \mathrm{~m}^{2}$, ambiente desprotegido, com leiras montadas nas dimensões de $1,0 \mathrm{~m} \times 1,0 \mathrm{~m} \times 0,60 \mathrm{~m}$ (comprimento x largura $\mathrm{x}$ altura). Na estabilização do aguapé efetuouse o aproveitamento de resíduos anteriormente descartados pela indústria, como os resíduos de suínos, gerados na higienização das pocilgas e limpeza dos caminhões que transportam os animais até a indústria, e as tripas celulósicas geradas durante o processo de industrialização da salsicha.

Utilizou-se quatro tratamentos distintos e duas repetições por tratamento, sendo: $\mathrm{T}_{1}-$ Aguapé (E. crassipes); $\mathrm{T}_{2}$ - Aguapé e dejeto suíno (7:1); $\mathrm{T}_{3}$ - Aguapé, dejeto suíno e terra $(7: 1: 0,67) ; \mathrm{T}_{4}-$ Aguapé, dejeto suíno e tripa celulósica $(7: 1: 0,67)$. Os resíduos foram misturados com base no volume de material in natura.
Após montagem das leiras de compostagem efetuou-se o monitoramento, durante um período de 90 dias, sendo avaliado semanalmente a temperatura ambiente e no interior das leiras e mensalmente a umidade, cinzas, nitrogênio total e carbono orgânico total. Efetuou-se o processo de aeração por meio de reviramento manual, o ciclo de reviramento foi quinzenal. Aos 90 dias mediu-se o volume das leiras para avaliação da redução do volume.

Determinou-se a constante de bioestabilização da matéria orgânica na compostagem para os quatro tratamentos, através da equação:

$$
\mathrm{Mb}=\text { Mbo. } \mathrm{e}^{-\mathrm{kt}} \text {. (LEITE; PAVINELLI, 1999) }
$$

em que:

$\mathrm{Mb}=$ massa do substrato transformada em um dado tempo $(\mathrm{g})$;

$\mathrm{Mbo}=$ massa do substrato aplicada em $\mathrm{t}_{\mathrm{o}}(\mathrm{g})$

$\mathrm{K}=$ constante de bioestabilização $\left(\mathrm{d}^{-1}\right)$.

O delineamento experimental adotado foi inteiramente ao acaso, sendo que para análise de variância (ANOVA) utilizou-se o Teste F, empregando o programa computacional denominado SISVAR e a determinação e ajuste da constante de bioestabilização foi efetuado por meio de regressão linear usando planilha eletrônica.

\section{Resultados e discussão}

A Tabela 1 mostra a caracterização dos diferentes substratos testados no processo de compostagem.

Tabela 1. Caracterização dos diferentes substratos testados no processo de compostagem.

\begin{tabular}{lccccccc}
\hline SUBSTRATO & $\begin{array}{c}\text { N total } \\
(\%)\end{array}$ & $\begin{array}{c}\mathbf{P} \text { total } \\
\mathbf{( \% )}\end{array}$ & $\begin{array}{c}\mathbf{K} \\
\mathbf{( \% )}\end{array}$ & $\begin{array}{c}\text { COT* } \\
\mathbf{( \% )}\end{array}$ & $\begin{array}{c}\text { UMIDADE } \\
\mathbf{( \% )}\end{array}$ & $\begin{array}{c}\text { SV* } \\
\mathbf{( \% )}\end{array}$ & $\mathbf{C : N}$ \\
Aguapé & 4,28 & 0,68 & 3,4 & 36,69 & 83,71 & 85,92 & 8,6 \\
Dejeto de suíno & 3,65 & 2,5 & 3,1 & 38,2 & 54,17 & - & 10,5 \\
Terra & 0,49 & 0,23 & 0,1 & 0,39 & 1,48 & 12,80 & 0,8 \\
Tripa celulósica & 0,17 & 0,097 & 0,1 & 44,02 & 5,96 & 99,89 & 259 \\
\hline
\end{tabular}

*COT: carbono orgânico total; SV: sólidos voláteis. 
Kawai e Grieco (1983) encontraram percentuais médios de $37 \%$ de carbono, 3,1\% de nitrogênio e $0,54 \%$ de fósforo em aguapés produzidos em lagoas experimentais. O'Brien (1980) apud Kawai e Grieco (1983) afirma que o percentual de carbono na composição química do aguapé, varia na faixa de 36,9 a 51,6\%; o nitrogênio de 1,56 a $3,94 \%$ e o fósforo de 0,31 a $0,89 \%$. Goyal, Dhull e Kapoor (2005), avaliando diferentes substratos para produção de compostos orgânicos, observaram para o jacinto d’água (aguapé), percentual de 41,8\% de carbono orgânico total (COT) e 2,31\% para nitrogênio total.

Os resultados obtidos durante o processo de compostagem podem ser visualizados na Tabela 2.

Tabela 2. Valores de nitrogênio total, carbono orgânico total, umidade e relação C:N aos 30,60 e 90 dias de compostagem.

\begin{tabular}{|c|c|c|c|c|c|c|c|c|c|c|c|c|c|}
\hline \multirow{2}{*}{\multicolumn{2}{|c|}{ TRATAMENTOS }} & \multicolumn{3}{|c|}{ N (\%) } & \multicolumn{3}{|c|}{$\mathrm{C}(\%)$} & \multicolumn{3}{|c|}{ Umidade (\%) } & \multicolumn{3}{|c|}{$C: N$} \\
\hline & & 30 & 60 & 90 & 30 & 60 & 90 & 30 & 60 & 90 & 30 & 60 & 90 \\
\hline \multirow[b]{2}{*}{$\mathrm{T}_{1}$} & 1 & 2,16 & 1,97 & 2,1 & 8,4 & 7 & 5 & 43,9 & 31,2 & 29 & 3,9 & 3,6 & 2,4 \\
\hline & 2 & 2,33 & 2,12 & 2,5 & 13,3 & 11 & 10 & 39,3 & 30,5 & 26,8 & 5,7 & 5,2 & 4 \\
\hline \multirow{2}{*}{$\mathrm{T}_{2}$} & 1 & 0,8 & 1,17 & 1,1 & 9,2 & 8 & 6,5 & 30,2 & 28,4 & 27,2 & 11,5 & 6,8 & 5,9 \\
\hline & 2 & 0,62 & 1,88 & 1,9 & 7,4 & 8 & 7 & 48,4 & 35,7 & 28,9 & 11,9 & 4,3 & 3,7 \\
\hline \multirow[b]{2}{*}{$\mathrm{T}_{3}$} & 1 & 0,36 & 0,83 & 0,92 & 4,1 & 4 & 4,5 & 30,9 & 31,5 & 31,6 & 11,4 & 4,8 & 4,9 \\
\hline & 2 & 0,28 & 1,25 & 1,2 & 4,3 & 2,5 & 2,7 & 32,2 & 29,3 & 20,4 & 15,4 & 2 & 2,3 \\
\hline \multirow{2}{*}{$\mathrm{T}_{4}$} & 1 & 0,32 & 0,92 & 0,87 & 5 & 5 & 4,5 & 24,3 & 29,8 & 29,1 & 15,6 & 5,4 & 5,2 \\
\hline & 2 & 0,57 & 1,28 & 1,17 & 5,5 & 11 & 10 & 32,5 & 30,9 & 21 & 9,6 & 8,6 & 8,5 \\
\hline
\end{tabular}

Observa-se pela Tabela 2, que o conteúdo de carbono diminuiu com o progresso da decomposição, o que de acordo com Bidone e Pavinelli (1999) ocorre principalmente devido às perdas de carbono como dióxido de carbono. Para Kiehl (1998), o carbono é utilizado como fonte de energia e o nitrogênio como fonte básica para reprodução e crescimento celular.

O Nitrogênio total nos tratamentos $\left(T_{2}, T_{3}\right.$ e $\left.T_{4}\right)$ aumentou com o tempo. No caso do tratamento $\left(\mathrm{T}_{1}\right)$, somente aguapé, observou-se uma diminuição do $\mathrm{N}$ total nas amostras coletadas após 30 dias. Segundo Goyal, Dhull e Kapoor (2005) a diminuição do nitrogênio nos estágios iniciais de decomposição ocorre devido às perdas de nitrogênio na forma de amônia, que dependem do tipo de material e da proporção C:N. De acordo com Reddy et al. (1979) e Sanchez-Monedero et al. (2001) apud Goyal, Dhull e Kapoor (2005) a compostagem de materiais com baixa proporção $\mathrm{C}: \mathrm{N}$ resulta em maiores perdas de $\mathrm{N}$ que a de materiais com alta proporção C:N. A relação adequada fica em torno de 30:1 (PEREIRA NETO, 1996), e de 25:1 a 30:1 (KIEHL, 1998).

Com relação à umidade do composto orgânico obtido, em média os quatro tratamentos obtiveram bons percentuais de umidade, que de acordo com Kiehl e Porta (1980), deve variar de 25 a 35\%.

Durante o experimento foi efetuado monitoramento semanal da temperatura média interna das pilhas de compostagem e da temperatura média ambiente, observou-se que houve aumento inicial de temperatura em todos os tratamentos, sendo os valores superiores aos da temperatura ambiente a partir da segunda semana de compostagem. Os valores de temperatura mantiveram-se entre $20 \mathrm{e}$ $30^{\circ} \mathrm{C}$ ficando abaixo do ideal para o processo de decomposição, segundo Kiehl (1998) a temperatura, principalmente na etapa que vai de 0 a 30 dias, deve ser de até $55^{\circ} \mathrm{C}$. Provavelmente este fato tenha ocorrido devido ao tamanho das partículas submetidas à decomposição, bem como devido ao tamanho das leiras de compostagem. 
Cezar et al. (2005) em ensaios de compostagem efetuados com plantas aquáticas por um período de 90 dias, observaram temperaturas que variaram de aproximadamente $10^{\circ} \mathrm{C}$ a $30^{\circ} \mathrm{C}$. Goyal, Dhull e Kapoor (2005) em ensaios de compostagem efetuados com diferentes resíduos orgânicos, observaram uma temperatura inicial de 28 a $30^{\circ} \mathrm{C}$ e após 14 dias, no tratamento com aguapé (jacinto d'água), observaram temperatura de $46^{\circ} \mathrm{C}$, que depois caiu gradativamente.

Embora não tenha se observado nas leiras de compostagem valores ideais de temperatura para o processo de decomposição, nos quatro tratamentos o material foi compostado como demonstrado pelos dados de redução da relação C:N (Tabela 2). De acordo com Goyal, Dhull e Kapoor (2005) não existe um único parâmetro que pode ser usado como indicador de maturidade de uma grande variedade de compostos, preparados com diferentes materiais, porém uma relação que é freqüentemente utilizada como índice de maturidade é a proporção $\mathrm{C}: \mathrm{N}$, quando um resíduo é compostado geralmente existe uma redução nesta proporção.

Após 90 dias de compostagem observou-se redução de aproximadamente $90 \%$ no volume do tratamento $\mathrm{T}_{1}$, de $88 \%$ nos volumes dos tratamentos $\mathrm{T}_{2}$ e $\mathrm{T}_{4}$ e de $67 \%$ no tratamento $\mathrm{T}_{3}$. Cezar et al. (2005) em ensaios de compostagem efetuados com plantas aquáticas em leiras de $2,5 \mathrm{~m}^{3}$ observaram redução de $80 \%$ do volume, em um período de 90 dias.

Equações exponenciais foram ajustadas aos dados de concentração de carbono orgânico total e nitrogênio total na compostagem durante o processo de bioestabilização da matéria orgânica, estando os melhores ajustes apresentados na Tabela 3.

Tabela 3. Equações exponenciais ajustadas aos dados de carbono orgânico total e nitrogênio total para os quatro tratamentos distintos.

\begin{tabular}{lcrrr}
\hline TRATAMENTO & T1 & T2 & T3 & \multicolumn{1}{c}{ T4 } \\
COT & $\mathrm{COT}=36,69 \cdot \mathrm{e}^{-0,0165 \mathrm{t}}$ & $\mathrm{COT}=36,87 \cdot \mathrm{e}^{-0,0171 \mathrm{t}}$ & $\mathrm{COT}=34,06 \cdot \mathrm{e}^{-0,0233 \mathrm{t}}$ & $\mathrm{COT}=37,43 \cdot \mathrm{e}^{-0,0151 \mathrm{t}}$ \\
Ntotal & $\mathrm{NT}=4,28 \cdot \mathrm{e}^{-0,00651 \mathrm{t}}$ & $\mathrm{NT}=4,2 \cdot \mathrm{e}^{-0,00771 \mathrm{t}}$ & $\mathrm{NT}=3,91 \cdot \mathrm{e}^{-0,00911 \mathrm{t}}$ & $\mathrm{NT}=3,89 \cdot \mathrm{e}^{-0,01041 \mathrm{t}}$ \\
\hline
\end{tabular}

A análise de variância aplicada às equações ajustadas para predizer a concentração de carbono orgânico total e de nitrogênio total não diferiu ao nível de $5 \%$ de probabilidade, pois os valores de $\mathrm{F}$ calculados para as concentrações de COT e de $\mathrm{N}$ total foram menores que o $\mathrm{F}$ tabelado, a um nível de 95\% de confiança $(6,59)$. Esses resultados permitiram concluir que os quatro tratamentos apresentaram constante de bioestabilização de primeira ordem estatisticamente iguais, para concentração de COT e NT nas leiras de compostagem. As constantes de bioestabilização médias para COT e NT foram: $\mathrm{K}_{\mathrm{COT}}$ $=1,8 \times 10^{-2} \mathrm{dia}^{-1}$ e $\mathrm{K}_{\mathrm{NT}}=0,8 \times 10^{-3} \mathrm{dia}^{-1}$. Matos et al. (1998), trabalhando com alguns resíduos orgânicos e utilizando águas residuárias da suinocultura como fonte de nitrogênio, obtiveram uma constante de média de estabilização do material orgânico de $0,3673 \times 10^{-2}$ dia $^{-1}$ para um período de 90 a 133 dias.

A Figura 1 apresenta as curvas de concentração de COT e de $\mathrm{N}$ total em função do tempo, respectivamente. 

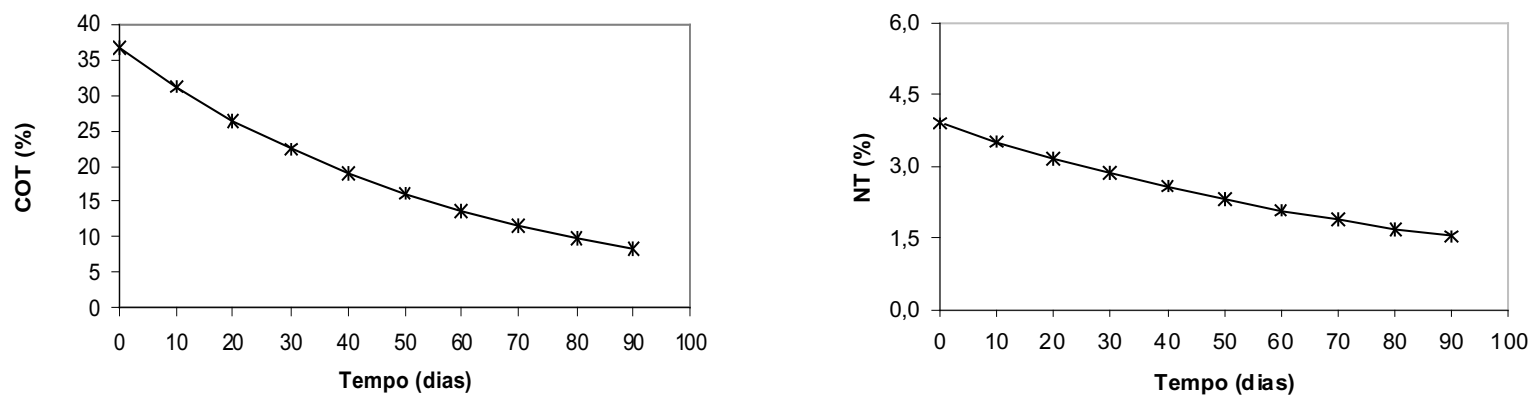

Figura 1. Curvas da concentração de carbono orgânico total e de nitrogênio total durante o período de compostagem (90 dias)

\section{Conclusões}

De acordo com os resultados obtidos conclui-se que:

Não houve diferença entre os quatro tratamentos avaliados no período de 90 dias. As taxas de bioestabilização média para o carbono orgânico total e para o nitrogênio total foram, respectivamente, $1,8 \times 10^{-2} \mathrm{dia}^{-1}$ e $0,8 \times 10^{-3} \mathrm{dia}^{-1}$.

O tratamento T4 foi considerado o mais indicado para a compostagem de toda a biomassa removida do sistema, por agregar valor ao resíduo de suínos e à tripa celulósica, resíduos estes que acarretam ônus à empresa no seu descarte.

\section{Referências}

BIDONE, F. R. A.; PAVINELLI, J. Conceitos básicos de resíduos sólidos. 1. ed. São Carlos: EESC/USP, 1999.

CEZAR,V. R. S.; VILLAS BOAS, R. L.; CORREAA, M. R.; NEGRISOLI, E.; VELINI, E. D. Avaliação da degradação de macrófitas aquáticas descartadas em ambiente protegido. Planta Daninha, Rio de Janeiro, v. 23, n. 2, p. 255-261, 2005.

GRADY, C. P. L.; DAIGGER, G. T.; LIM, H. C. Biological wastewater treatment. 2. ed. New York: Marcel Dekker, 1999.

GOYAL, S.; DHULL, S. K.; KAPOOR, K. K. Chemical and biological changes during composting of different organic wastes and assessment of compost maturity. Bioresource Technology, Essex, v. 96, n. 14, p. 15841591, 2005.
KAWAI, H.; GRIECO, V. M. Utilização do aguapé para tratamentos de esgoto doméstico. Estabelecimento de critérios de dimensionamento de lagoa de aguapé e abordagem de alguns problemas operacionais. Revista $D A E$, São Paulo, v. 44, n. 135, p. 79-90, 1983.

KIEHL, E. J. Manual de compostagem: maturação e qualidade do composto. Piracicaba: E. J. Kiehl, 1998.

KIEHL, E. J.; PORTA, A. Análises de lixo e compostos. 1. ed. Piracicaba: ESALQ, 1980.

LEITE, V. D.; PAVINELLI, J. Comportamento dos sólidos totais no processo de digestão anaeróbia de resíduos sólidos urbanos e industriais. Revista Engenharia Agricola e Ambiental, Campina Grande, v. 3, n. 2, p. 229232, 1999.

LIMA, M. R.; TAFFAREL, A. D.; REISSMANN, C. B.; SILVA, A. G. Crescimento e absorção de alguns elementos químicos em aguapé, alface da água e lentilha da água, no período de inverno, em Pinhais-PR. In: SEMINÁRIO DO PROJETO INTERDISCIPLINAR SOBRE EUTROFIZAÇÃO DE ÁGUAS DEABASTECIMENTO PÚBLICO NA BACIA DO ALTÍSSIMO IGUAÇU, 4., 2003, Curitiba. Resumos... Curitiba: Companhia de Saneamento do Paraná, 2003. p. 1-3.

MATOS, A. T.; VIDIGAL, S. M.; SEDIYAMA, M. A. N.; GARCIA, N. C. P.; RIBEIRO, M. F. Compostagem de alguns resíduos orgânicos, utilizando-se águas residuárias da suinocultura como fonte de nitrogênio. Revista Brasileira de Engenharia Agrícola e Ambiental, Campina Grande, v. 2, n. 2, p. 199-203, 1998.

MORAES, A. J.; RODRIGUES, J. B.; NICODEM, M. F. M.; RODRIGUES, M; B. Remoção de fosforo com uso de macrófitas em lagoa facultativa de frigorífico. In: ENCONTRO NACIONAL DE DIFUSÃO TECNOLÓGICA, 1., 2002, Medianeira. Anais... Medianeira: ENDITEC, 2002, p. 62-64. 
PEREIRANETO, J.T.Manual de compostagem: processo de baixo custo. Belo Horizonte: UNICEF, 1996.

SUZEK, M.; SAMPAIO, S. C.; SANTOS, R. F.; NUNES, H. L. G. S.; GOMES, S. D.; MALLMANN, L. S. Uso de água residuária da suinocultura na bioestalização de resíduos verdes urbanos. Revista Engenharia Agrícola e Ambiental, Campina Grande, v. 9, p. 176-180, 2005. Suplemento.

WOLVERTON, B. C.; MCDONALD, R. The water hyacinth: From prolific pest to potencial provider. Ambio, Oslo, v. 8, n. 1, p. 2-9, 1979. 
